\title{
Morphology and Structures of Nearby Dwarf Galaxies
}

\author{
Mira Seo and H. B. Ann \\ Dept. of Earth Science, Pusan National University \\ 609-735 , Busan, Korea \\ email: mrseo@pusan.ac.kr, hbann@pusan.ac.kr
}

\begin{abstract}
We applied GALFIT and STARLIGHT to the $r$-band images and spectra, respectively, of $\sim 1,100$ dwarf galaxies to analyze the structural properties and stellar populations. In most cases, single component with $n=1 \sim 1.5$ well describes the luminosity distribution of dwarf galaxies. However, a large fraction of $\mathrm{dS} 0, \mathrm{dE}_{b c}$, and $\mathrm{dE}_{b l u e}$ galaxies show sub-structures such as spiral arms and rings. There is a bimodal distributions of stellar ages in dS0 galaxies. But other sub-types of dwarf galaxies show a single peak in the stellar distributions.
\end{abstract}

Keywords. galaxies, dwarf, structure, population

\section{Introduction}

Understanding dwarf galaxies is crucial in the mordern cosmology because they are most dominant populations in the universe. However, until recently, there is no detailed morphological studies of dwarf galaxies. Ann, Seo, \& Ha (2015) determined morphological types of the local galaxies $(z<0.01)$ using color images of the Sloan Digital Sky Survey (SDSS) DR7 distinguishing sub-types of dwarf elliptical-like galaxies. The present study aims at understanding the structural properties of dwarf elliptical-like galaxies using $r$-band SDSS images and spectral data.

\section{Data}

We used 1,100 dwarf elliptical-like galaxies in the catalog of Ann, Seo, \& Ha (2015). They divided dwarf elliptical-like galaxies into 5 sub-types: dwarf lenticular galaxies (dS0), dwarf elliptical galaxies (dE), blue-cored dwarf elliptical galaxies $\left(\mathrm{dE}_{b c}\right)$, blue dwarf elliptical galaxies $\left(\mathrm{dE}_{\text {blue }}\right)$, and dwarf spheroid galaxies (dSph). Among the 5 subtypes, dS0, dE, and dSph galaxies show the presence of nucleation. Along with the morphological types, we used the $r$-band images and spectra of dwarf elliptical-like galaxies to analyze the luminosity distributions and stellar populations.

\section{Methods}

We performed 2D-photometric decompositions of dwarf elliptical-like galaxies using GALFIT (Peng, Ho, Impey \& Rix 2002, 2010). Residual images are obtained by subtracting the single component model images from the observed galaxy images. We computed spectral synthesis models using STARLIGHT (Cid Fernandes et al. 2005) and obtained light-fraction population vectors $\left(\mathrm{x}_{j}\right)$ in 15 different age groups and 6 metallicity groups. We derived the local background density by the nth nearest neighbor technique. We used $\mathrm{n}=5, \Delta \mathrm{V}=500 \mathrm{~km} / \mathrm{s}$, and $\mathrm{M}^{*}=-15.24$. 


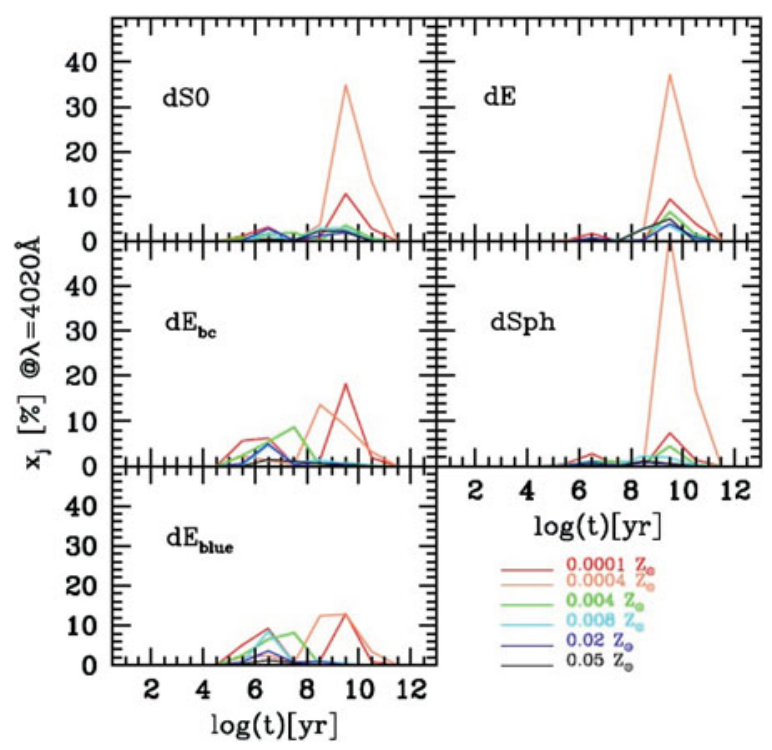

Figure 1. Age distributions in the population vector $(\mathrm{x}[\%])$ of dwarf elliptical-like galaxies, distinguished by their metallicity

\section{Results and Discussion}

There is no significant difference in the physical parameters, residual features and their environment between nucleated dwarfs $\left(\mathrm{dE}_{n}, \mathrm{dSph}_{n}, \mathrm{dS} 0_{n}\right)$ and un-nucleated dwarfs $\left(\mathrm{dE}_{u n}, \mathrm{dSph}_{u n}, \mathrm{dSO}_{u n}\right)$. There are a variety of features in the residual images such as lens, bar, and spiral arms. These features are more frequent in $\mathrm{dS} 0, \mathrm{dE}_{b c}$ and $\mathrm{dE}_{b l u e}$ galaxies. The residual images of $\mathrm{dE}_{\text {blue }}$ galaxies suggest that some $\mathrm{dE}_{\text {blue }}$ galaxies have nuclei which are difficult to be noticed due to their colors similar to their global colors. Half of dS0 galaxies show a variety of sub-structures in their residual images.

Fig. 1 shows the age distributions of dwarf elliptical-like galaxies sorted by the metallicities. The $\mathrm{dE}$, and $\mathrm{dSph}$ galaxies have mostly old populations while $\mathrm{dE}_{b c}$ and $\mathrm{dE}_{b l u e}$ galaxies have a mixture of populations, characterized by a bimodal distribution. The dS0 galaxies show intermediate distributions. Combined with metallicity distribution, old stellar populations are mostly metal poor but young stellar populations have similar fractions of metal poor and metal rich stars. It is of interest to see that age distributions of stars with intermediate metallicity $\left(\mathrm{z}=0.004 z_{\odot}\right)$ display bimodal distributions in dS0 galaxies while there are single peaks at old stellar populations in $\mathrm{dE}$ and $\mathrm{dSph}$ and at young stellar populations at intermediate age populations in $\mathrm{dE}_{b c}$ and $\mathrm{dE}_{b l u e}$.

\section{References}

Ann, Seo, Ha 2015, ApJS, 217, 27

Cid Fernandes, R., Mateus, A. , Sodre, L., Stasinska, G., \& Gomes, J. M. 2005, MNRAS, 358,363

Peng, C. Y., Ho, L. C., Impey, C. D., \& Rix, H.-W. 2002, AJ, 124, 266

Peng, C. Y., Ho, L. C., Impey, C. D., \& Rix, H.-W. 2010, AJ, 139, 2097 